\title{
Does Obesity Increase The Morbidity and Mortality in COVID-19 Patients? Evidence Based Clinical Review
}

\author{
Dr. Ajeet Subramaniam ${ }^{1 *}$, Dr. Rania Mansour Elsayed ${ }^{2}$
}

${ }^{1}$ Consultant Family Medicine, Primary Healthcare Corporation, Doha, Qatar

${ }^{2}$ Consultant Emergency Medicine, Primary Healthcare Corporation, Doha, Qatar

DOI: $10.36347 /$ sjams.2021.v09i02.014

| Received: 02.02.2021 | Accepted: 14.02.2021 | Published: 23.02.2021

*Corresponding author: Dr. Ajeet Subramaniam

Abstract

Obesity is a global disease. This study explores the links between obesity and morbidity and mortality in COVID-19. The coronavirus disease 2019 (COVID 19) is caused by a single stranded RNA virus. Recent data suggest that people with obesity are at increased risk of severe COVID-19. Obesity increases risk for hospitalization, ICU admission, Invasive Mechanical Ventilation requirement and death among patients with COVID-19. This study also highlights the pathways by which complications arise in severe acute respiratory syndrome coronavirus 2 (SARS-CoV-2). A hyperinflammatory reaction through the excessive release of cytokines, a condition known as "cytokine storm is responsible for increased morbidity and mortality. We also discuss how severe acute respiratory syndrome coronavirus 2 penetrates human cells through direct binding with angiotensin-converting enzyme 2 receptors on the cell surface. Angiotensin-converting enzyme 2 expression in adipose tissue is higher than that in lung tissue, which means that adipose tissue may be vulnerable to COVID-19 infection.

Keywords: Coronavirus disease -2019 (COVID-19), angiotensin-converting enzyme 2 (ACE2), SARS-CoV-2(severe acute respiratory syndrome-coronavirus -2), Body mass index, Intensive care; Invasive mechanical ventilation; Mortality; Obesity; Visceral adipose tissue. HOMA-IR (Homeostatic Model Assessment for Insulin Resistance), Mammalian target of rapamycin (mTOR).

Copyright $($ C) 2021 The Author(s): This is an open-access article distributed under the terms of the Creative Commons Attribution 4.0 International License (CC BY-NC 4.0) which permits unrestricted use, distribution, and reproduction in any medium for non-commercial use provided the original author and source are credited.

\section{INTRODUCTION}

This study first establishes Obesity as a risk factor for COVID-19 infection. Then it highlights the pathogenesis of complications that arise in coronavirus infection. Subsequently we discuss important characteristics of Obese patients with COVID-19 infection like increased contagiousness due to prolonged virus shedding. In the end we discuss some suggestions to manage the increased risks that Obese patients present in COVID-19 infection.

\section{Obesity as Risk factor for severity in COVID-19 infection}

Hussain et al., [1] described obesity as a global disease and tried to explore the links between obesity and mortality in COVID-19. They studied data from various research studies and concluded that patients with obesity are at high risk of mortality from COVID-19 infection.
Stefan et al., [2] also stated that preliminary data suggest that people with obesity are at increased risk of severe COVID-19. Patients have increased risk of pneumonia and difficulties of intubation and mask ventilation, the risk of death. It is better to estimate the risk of complications in patients with COVID-19 by evaluation of standard hospital parameters (such as the Sequential Organ Failure Assessment, d-dimer and proinflammatory markers) and by the measurement of anthropometrics and metabolic parameters. These parameters include BMI, waist and hip circumferences and levels of glucose and insulin. The latter two parameters can be used for the estimation of insulin resistance, for example by calculation of the HOMA-IR (Homeostatic Model Assessment for Insulin Resistance). Knowledge about insulin resistance is important, because it is among the strongest determinants of impaired metabolic health, cardiac dysfunction and CVD-related mortality. Such measurements might be useful both in a primary care setting and, in a hospital, setting to assess the risk of a complicated course of disease in patients with a positive 
SARS-CoV-2 test. In conclusion, while it is widely recognized that the presence of comorbidities such as hypertension, diabetes mellitus and CVD is associated with a more severe course of COVID-19, obesity has hardly been investigated so far. Obesity is a main risk factor for these comorbidities and more generally for impaired metabolic health (such as dyslipidaemia and insulin resistance) and is also linked to an increased risk of pneumonia. Measurement of anthropometric characteristics and metabolic parameters is crucial to better estimate the risk of complications in patients with COVID-19.

Busetto., et al., [3] also described that the clinical manifestations of coronavirus disease (COVID19) run from asymptomatic disease to severe acute respiratory syndrome. Older age and comorbidities are associated to more severe disease. A role of obesity is suspected. Patients with overweight and obesity admitted in a medical ward for severe acute respiratory syndrome coronavirus 2-related pneumonia, despite their younger age, required more frequently assisted ventilation and access to intensive or semi-intensive care units than normal weight patients.

Cai et al., [4] stated that the patients with obesity are at increased risk of exacerbations from viral respiratory infections. They observed that obese patients had increased odds of progressing to severe COVID-19. As the severe acute respiratory syndrome coronavirus 2 may continue to spread worldwide, clinicians should pay close attention to obese patients, who should be carefully managed with prompt and aggressive treatment.

Huang et al., [5] studied to explore the effects of obesity with the risk of severe disease among patients with COVID-19. Body mass index (BMI) and degree of visceral adipose tissue (VAT) accumulation were used as indicators for obesity status. Clinical outcomes of severe COVID-19 included hospitalization, a requirement for treatment in an intensive care unit (ICU), invasive mechanical ventilation (IMV), and mortality. Obesity increases risk for hospitalization, ICU admission, IMV requirement and death among patients with COVID-19. Further, excessive visceral adiposity appears to be associated with severe COVID19 outcomes. These findings emphasize the need for effective actions by individuals, the public and governments to increase awareness of the risks resulting from obesity and how these are heightened in the current global pandemic.

De Siqueira et al., [6] also aimed to assess whether there was an association between obesity and higher levels of hospitalization, poor outcomes and mortality due to the disease of novel coronavirus (COVID-19). In their study obesity and overweight were represented an unfavourable factor for infection of novel coronavirus, where the higher the BMI the worse the outcomes. This occurred by worsening the infection itself, as well as increasing the prevalence of hospitalizations, worst outcomes and greater lethality; especially when co-occurring with other chronic conditions and in the elderly as well. Given this evidence, greater attention is suggested to the obese and overweight population in the face of the current pandemic.

\section{Pathogenesis of complications in Obese patients with COVID-19 infection}

Korakas et al., [7] described the pathogenesis of complications in obese patients. They pointed out a strong association between obesity and the severity of COVID-19 infection, even in the absence of other comorbidities. After infecting the host cells, severe acute respiratory syndrome coronavirus 2 (SARS-CoV2) may cause a hyperinflammatory reaction through the excessive release of cytokines, a condition known as "cytokine storm," while inducing lymphopenia and a disrupted immune response. Obesity is associated with chronic low-grade inflammation and immune dysregulation, but the exact mechanisms through which it exacerbates COVID-19 infection are not fully clarified. The production of increased amounts of cytokines such as TNFa, IL-1, IL-6, and monocyte chemoattractant protein (MCP-1) lead to oxidative stress and defective function of innate and adaptive immunity, whereas the activation of NOD-like receptor family pyrin domain containing 3 (NLRP3) inflammasome seems to play a crucial role in the pathogenesis of the infection. Endothelial dysfunction and arterial stiffness could favour the recently discovered infection of the endothelium by SARS-CoV2 , whereas alterations in cardiac structure and function and the prothrombotic microenvironment in obesity could provide a link for the increased cardiovascular events in these patients. The successful use of antiinflammatory agents such as IL-1 and IL-6 blockers in similar hyperinflammatory settings, like that of rheumatoid arthritis, has triggered the discussion of whether such agents could be administrated in selected patients with COVID-19 disease.

Petrakis et al., [8] also described Coronaviruses ( $\mathrm{CoV})$ as a group of viruses that cause infections in the human respiratory tract, which can be characterized clinically from mild to fatal. The severe acute respiratory syndrome coronavirus 2 (SARS-CoV-2) is the virus responsible. A growing body of evidence connects obesity with COVID-19 and a number of mechanisms from immune system activity attenuation to chronic inflammation are implicated. Lipid peroxidation creates reactive lipid aldehydes which in a patient with metabolic disorder and COVID-19 will affect its prognosis. Finally, pregnancy-associated obesity needs to be studied further in connection to COVID-19 as this infection could pose high risk both to pregnant women and the foetus. 
Ajeet Subramaniam \& Rania Mansour Elsayed; Sch J App Med Sci, Feb, 2021; 9(2): 248-252

Sanchis-Gomar et al., [9] described that Obesity has reached epidemic proportions in the United States and in much of the westernized world, contributing to considerable morbidity. Several of these obesity-related morbidities are associated with greater risk for death with coronavirus disease 2019 (COVID19). Severe acute respiratory syndrome coronavirus 2 penetrates human cells through direct binding with angiotensin-converting enzyme 2 receptors on the cell surface. Angiotensin-converting enzyme 2 expression in adipose tissue is higher than that in lung tissue, which means that adipose tissue may be vulnerable to COVID19 infection. Obese patients also have worse outcomes with COVID-19 infection, including respiratory failure, need for mechanical ventilation, and higher mortality. Clinicians need to be more aggressive when treating obese, especially severely obese, patients with COVID19 infection.

Bolourian et al., [10] discussed that the mechanisms by which obesity increases the rate/mortality of COVID-19 need to be comprehensively understood. Possible mechanisms include associated comorbidities and enhanced virus entry through the human angiotensin- converting enzyme 2 (ACE2). ACE2 is the putative receptor for coronavirus entry into host cells. In adipose tissues, ACE2 expression levels exceed those expressed in the lung. Individuals with obesity present an increased volume of adipose tissues, and consequently higher ACE2 levels, which could increase their susceptibility to COVID- 19. Viruses largely rely on the host cell translation machinery, exploiting the translational apparatus of the infected cell to express viral proteins. Certain pathways/molecules, hyperactivated in obese hosts and shared with those in coronaviruses, could further rationalize the association of obesity with COVID- 19.

Mammalian target of rapamycin (mTOR) pathway is composed of a series of molecules highly conserved in eukaryotes during evolution, and regulates translation, protein and lipid synthesis, proliferation, and growth in response to nutrient availability.

The mTOR pathway integrates nutrient signals to regulate the translation initiation of mRNAs crucial for biosynthesis. Upon feeding, the synthesis machinery and mTOR pathway are triggered within $30 \mathrm{~min}$ in the liver, remain elevated for $90 \mathrm{~min}$, and return to baseline after $120 \mathrm{~min}$. However, following nutrient starvation, the mTOR pathway is downregulated to adjust cellular metabolism for survival. Both in- vitro and in- vivo studies have revealed the association between obesity and the mTOR pathway._Notably, obesity and overnutrition trigger chronic hyperactivation of mTOR activity in multiple tissues. Indeed, targeting the mTOR pathway has been suggested as a treatment for obesity. In conclusion, mTOR pathway hyperactivation and enhanced translational apparatus in obesity/overnutrition might provide an ideal platform for coronavirus replication, demonstrating translation dependent on the host cells.

Iannelli et al., [11] described that the angiotensin-converting enzyme (ACE) 2 is targeted by SARS-CoV 2. In parallel, obesity appears as the main risk factor for severe forms of COVID-19. The RAAS and obesity are closely related, leading to the aggravation of the disease.

It is of paramount importance to note that weight loss, even modest, reverses the imbalance of the RAAS at the adipose tissue as well as at the systemic levels. Additionally, the adipose tissue may host in obese individuals a larger viral load behaving as a reservoir and giving a higher potential for diffusing the virus and contaminating other individuals.

Upon that, it may be speculated that individuals with a history of bariatric surgery may have a reduced risk of the severe form of COVID-19 disease compared with obese individuals without bariatric surgery. While this remains speculative and in light of these pieces of evidence, the fact that bariatric surgery is currently being held still with thousands of patients waiting for their surgery should be questioned.

\section{Important characteristics of Obese patients with COVID-19 infection \\ Luzi et al., [12] described that the basal} hormone milieu, defective response of both innate and adaptive immune system and sedentariness are major determinants in the severity of influenza viral infection in obese patients. Being overweight not only increases the risk of infection and of complications for the single obese person, but a large prevalence of obese individuals within the population might increase the chance of appearance of more virulent viral strain, prolongs the virus shedding throughout the total population and eventually might increase overall mortality rate of an influenza pandemic. Evidence from previous influenza pandemics suggests the following interventions aimed at improving immune response: (1) lose weight with a mild caloric restriction; (2) include AMPK activators and PPAR gamma activators in the drug treatment for obesity associated with diabetes; and (3) practice mild-to-moderate physical exercise. Due to prolonged viral shedding, quarantine in obese subjects should likely be longer than normal weight individuals.

Popkin et al., [13] pointed out that individuals with obesity are linked with large significant increases in morbidity and mortality from COVID-19. There are many mechanisms that jointly explain this impact. A major concern is that vaccines will be less effective for the individuals with obesity. 
Petrova et al., [14] suggest that obesity is a risk factor for more severe coronavirus disease. They proposed biological mechanisms that could explain both the higher risk observed and the possible higher contagiousness of people with obesity. They considered implications of the pandemic for people with obesity in relation to difficulties in managing hospitalized patients, implications of confinement for the control and treatment of obesity, and the stigma people with obesity suffer, that could increase should the relationship between obesity and COVID-19 be confirmed. Understanding the role of obesity in COVID-19 should be a public health priority, given the high prevalence of this condition in our country.

\section{Management options}

El-Missiry et al., [15] stated that severe acute respiratory syndrome coronavirus 2 (SARS-CoV-2), is a highly pathogenic virus that affects the respiratory system, produces an inflammatory storm that causes lung damage and respiratory dysfunction. It infects humans of all ages. The Covid-19 takes a more severe course in individuals with chronic metabolic diseases such as obesity, diabetes mellitus, and hypertension. This category of persons exhibits weak immune activity and decreased levels of endogenous antioxidants. Melatonin is a multifunctional signalling hormone synthesized and secreted primarily by the pineal gland. It is a potent antioxidant with immunomodulatory action and has remarkable anti-inflammatory effects under a variety of circumstances. Regarding Covid-19 and metabolic syndrome, adequate information about the relationship between these two comorbidities is required for better management of these patients. Since Covid-19 infection and complications involve severe inflammation and oxidative stress in people with obesity and diabetes, we anticipated the inclusion of melatonin, as powerful antioxidant, within proposed treatment protocols. In this context, melatonin is a potential and promising agent to help overcome Covid19 infection and boost the immune system in healthy persons and obese and diabetic patients.

The Lancet Diabetes Endocrinology [16] discussed obesity and COVID-19 in their editorial. They pointed out that the on July 27 th, 2020, the UK government released the new national strategy for tackling obesity. In big bold font, the heading of the official press release urges people to "Lose weight to beat COVID-19 and protect the NHS".

In the first 4 months of the pandemic, England witnessed the greatest rate of excess deaths in Europe. According to a New York Times analysis, between March 14 and July 17, 2020, the UK has had 62600 more deaths, a $31 \%$ increase, compared with the same period last year.

Obesity, along with other chronic conditions such as diabetes, is a recognised risk factor for severe clinical outcomes of COVID-19. The new obesity strategy, published alongside the 'Better Health' campaign, led by Public Health England, intends to restrict advertisements and promotions of unhealthy foods, improve nutritional labelling of foods and drinks in restaurants and stores, and expand weight management services.

The ambition for a healthier nation, during and beyond COVID-19, is to be praised. However, at a time where our economies are fragile, bans on food promotions and advertisements could also result in higher prices and growing inequalities, which themselves contribute to obesity and poor health outcomes. Equally troubling, by targeting almost exclusively the obesogenic environment, and only a small part of it, the new policies fail to take into account the intricacy of biological, societal, and psychological factors that underpin obesity.

The suggestion that it is necessary to lose weight to "reduce pressure on doctors and nurses in the NHS, and free up their time to treat other sick and vulnerable patients", communicated in the policy document, is also one of the most glaring examples of health promotion strategies that draw on guilt and shame. Past Public Health history has shown that such campaigns are ineffective and even detrimental.

The COVID-19 culture has become a blame culture. The obesity rates in England are concerning, but they are not the main culprit for the nation's high COVID-19 death toll. Let's not forget that people with obesity are vulnerable patients too.

\section{CONCLUSION}

Obesity, along with other chronic conditions such as diabetes, is a recognised risk factor for severe clinical outcomes of COVID-19. Severe acute respiratory syndrome coronavirus 2 penetrates human cells through direct binding with angiotensin-converting enzyme 2 receptors on the cell surface. Angiotensinconverting enzyme 2 expression in adipose tissue is higher than that in lung tissue, which means that adipose tissue may be vulnerable to COVID-19 infection. SARS-CoV-2 causes a hyperinflammatory reaction through the excessive release of cytokines, a condition known as "cytokine storm." These pathologic mechanisms are responsible for increased morbidity and mortality of obese patients with COVID-19 infection.

\section{REFERENCES}

1. Hussain A, Mahawar K, Xia Z, Yang W, Shamsi EH. Obesity and mortality of COVID-19. Metaanalysis. Obesity research \& clinical practice. 2020 Jul 9.

2. Stefan N, Birkenfeld AL, Schulze MB, Ludwig DS. Obesity and impaired metabolic health in patients 
with COVID-19. Nature Reviews Endocrinology. $2020 \mathrm{Jul} ; 16(7): 341-2$.

3. Busetto L, Bettini S, Fabris R, Serra R, Dal Pra C, Maffei P, Rossato M, Fioretto P, Vettor R. Obesity and COVID- 19: an Italian snapshot. Obesity. 2020 Sep;28(9):1600-5.

4. Cai Q, Chen F, Wang T, Luo F, Liu X, Wu Q, He Q, Wang Z, Liu Y, Liu L, Chen J. Obesity and COVID-19 severity in a designated hospital in Shenzhen, China. Diabetes care. 2020 Jul 1;43(7):1392-8.

5. Huang Y, Yao LU, Huang YM, Min WA, Wei LI, Yi SU, Hai-Lu ZH. Obesity in patients with COVID-19: a systematic review and meta-analysis. Metabolism. 2020 Sep 28:154378.

6. de Siqueira JV, Almeida LG, Zica BO, Brum IB, Barceló A, de Siqueira Galil AG. Impact of obesity on hospitalizations and mortality, due to COVID19: a systematic review. Obesity research \& clinical practice. 2020 Jul 23.

7. Korakas E, Ikonomidis I, Kousathana F, Balampanis K, Kountouri A, Raptis A, Palaiodimou L, Kokkinos A, Lambadiari V. Obesity and COVID-19: immune and metabolic derangement as a possible link to adverse clinical outcomes. American Journal of PhysiologyEndocrinology and Metabolism. 2020 Jul 1;319(1):E105-9.

8. Petrakis D, Margină D, Tsarouhas K, Tekos F, Stan M, Nikitovic D, Kouretas D, Spandidos DA, Tsatsakis A. Obesity-a risk factor for increased COVID-19 prevalence, severity and lethality. Molecular medicine reports. 2020 Jul 1;22(1):9-19.
9. Sanchis-Gomar F, Lavie CJ, Mehra MR, Henry BM, Lippi G. Obesity and outcomes in COVID-19: when an epidemic and pandemic collide. InMayo Clinic Proceedings 2020 May 19. Elsevier.

10. Bolourian A, Mojtahedi Z. Obesity and COVID19: The mTOR pathway as a possible culprit. Obesity Reviews. 2020 Sep 1.

11. Iannelli A, Favre G, Frey S, Esnault V, Gugenheim J, Bouam S, Schiavo L, Tran A, Alifano $\mathrm{M}$. Obesity and COVID-19: ACE 2, the missing tile. Obesity Surgery. 2020 Nov;30:4615-7.

12. Luzi L, Radaelli MG. Influenza and obesity: its odd relationship and the lessons for COVID-19 pandemic. Acta diabetologica. 2020 Jun;57(6):75964.

13. Popkin BM, Du S, Green WD, Beck MA, Algaith T, Herbst CH, Alsukait RF, Alluhidan M, Alazemi $\mathrm{N}$, Shekar M. Individuals with obesity and COVID- 19: A global perspective on the epidemiology and biological relationships. Obesity Reviews. 2020 Nov;21(11):e13128.

14. Petrova D, Salamanca-Fernández E, Barranco MR, Pérez PN, Moleón JJ, Sánchez MJ. La obesidad como factor de riesgo en personas con COVID-19: posibles mecanismos e implicaciones. Atención Primaria. 2020 Aug 1;52(7):496-500.

15. El-Missiry MA, El-Missiry ZM. Melatonin is a potential adjuvant to improve clinical outcomes in individuals with obesity and diabetes with coexistence of Covid-19. European journal of pharmacology. 2020 Jun 30:173329.

16. Diabetes TL. Obesity and COVID-19: Blame isn't a strategy. The Lancet. Diabetes \& Endocrinology. 2020 Aug 7. 